\title{
Morfometria e mortalidade em pupas e adultos de Brassolis astyra (Nymphalidae, Morphinae)
}

\author{
Geraldo Salgado-Neto \& Rocco Alfredo Di Mare
}

Departamento de Biologia, Centro de Ciências Naturais e Exatas, Universidade Federal de Santa Maria, 97119-900 Santa Maria, RS, Brasil. (gsalgado@bol.com.br; ram13@terra.com.br)

\begin{abstract}
Morphometry and mortality in pupae and adults of Brassolis astyra (Nymphalidae, Morphinae). The data presented are based on Brassolis astyra Godart, 1824 individuals sampled randomly in four locations of the State of Rio Grande do Sul, Brazil: Tupanciretã, São Martinho da Serra, Santa Maria - two places. Except in São Martinho da Serra, the data were obtained in urban areas, on Syagrus romanzoffianum palms (Palmaceae). The following parameters were investigated: egg laying number, hatching eggs index, number of miscarried eggs, eggs positioning on the host plant, sex ratio, parasitoid index, emergency pupa-adult frequency, longevity of pupae and adult stages, mass and volume of the pupae and morphometric measurements of pupae and adults' wings. Of eggs collected in Tupanciretã, $46 \%$ hatched, $43 \%$ had been parasitized and $11 \%$ were miscarried. On the other hand, in São Martinho da Serra only $4 \%$ of the eggs collected hatched, $95 \%$ had been parasitized and $1 \%$ were miscarried. In the other localities no eggs were found. Based on adults emerged from the pupae, it was verified that $77 \%$ of the males and females presented a normal morphology (viable) and $23 \%$ were malformed. The differences in the average values estimated for pupal longevity among the study sites, were statistically significant. The average frequency of adults was 0.47 . The data obtained showed a sexual dimorphism in size (weigh) and form (volume) of the pupae. This suggests that the size and the form of the pupae can determine the adults' viability. Such effect could be associated with a stabilizing selection, where would be associated to optima values average of weigh and form. Although the origin of the sampled individuals varied, the parameters that determine the size and shape are in the same direction when comparing the two sexes. Data on longevity and mortality suggest that the estimated values can be influenced by the variability and ecology of the population from which the larvae were sampled.
\end{abstract}

KEYWORDS. Butterflies, sexual dimorphism, urban ecology, longevity, sex ratio.

RESUMO. Os dados analisados são provenientes de indivíduos de Brassolis astyra Godart, 1824 amostrados em quatro localidades do Rio Grande do Sul, Brasil: Tupanciretã, São Martinho da Serra, Santa Maria - dois locais. Exceto em São Martinho da Serra, área natural, as demais coletas foram realizadas em áreas urbanas: todas em Syagrus romanzoffianum (Palmaceae). Os parâmetros analisados foram: número de ovos por postura, índice de eclosão de ovos, frequência de parasitoidismo, número de ovos malogrados, posição das posturas na planta hospedeira, proporção sexual, taxa de emergência de pupas e adultos, longevidade das pupas e dos adultos, massa e volume das pupas, e morfometria das pupas e das asas a partir de marcos anatômicos. Dos ovos coletados em Tupanciretã, área urbana, $46 \%$ eclodiram, $43 \%$ foram parasitados e 11\% malograram. Entretanto dos ovos coletados em São Martinho da Serra, área natural, $4 \%$ eclodiram, $95 \%$ foram parasitados e $1 \%$ malograram. Nas demais localidades não foram encontradas posturas. Com base nos adultos emergidos a partir das pupas verificou-se que $77 \%$ dos machos e das fêmeas apresentaram uma morfologia normal (viável) e $23 \%$ eram malformadas. As diferenças entre os valores médios obtidos para longevidade das pupas entre as localidades estudadas são estatisticamente significantes. A taxa média de emergência de adultos foi de 0,47. Os resultados obtidos mostram um dimorfismo sexual de tamanho (peso) e forma (volume) entre as pupas. Sugerem, também, que o tamanho e a forma das pupas podem determinar a viabilidade dos adultos. Tal fato pode estar associado a uma seleção estabilizadora, cujo peso e forma estariam associados a valores médios ótimos. Embora a procedência dos indivíduos analisados seja de diferentes locais os parâmetros obtidos que determinam o tamanho e a forma estão na mesma direção quando comparamos ambos os sexos. Os dados referentes à longevidade e à mortalidade sugerem que os valores estimados podem ser influenciados pela variabilidade e ecologia da população de onde as larvas foram retiradas.

PALAVRAS-CHAVE. Borboletas, dimorfismo sexual, ecologia urbana, longevidade, proporção sexual.

As borboletas do gênero Brassolis Fabricius, 1807 constituem um grupo cujo voo da maioria das espécies ocorre, principalmente, ao anoitecer e ao amanhecer. Em quase todas as espécies de lepidópteros, os adultos usam a espirotromba para se alimentar de néctar ou de frutos fermentados, exudações de plantas e animais em decomposição. No entanto, Brassolis, tem a espirotromba atrofiada e não se alimenta na fase adulta (RUSzczYK \& CARValho, 1993). Portanto, a viabilidade e reprodução dos adultos dependem da qualidade e quantidade do alimento disponível durante os estágios imaturos.

Para o Rio Grande do Sul existem registros de doze espécies de palmeiras atacadas por Brassolinae (BiEZAnKo, 1960a; Link et al., 1980). Segundo Link \& Alvarez Filho (1979), das quatorze espécies de palmeiras registradas para o estado somente em Chrysolidocarpus lutescens (Palmaceae) areca-bambu, não foi constatado ataque por brassolíneos. Há registros de ataque por Brassolis astyra Godart, 1824 em doze destas espécies. Entre as palmeiras investigadas a mais atacada foi Syagrus romanzoffianum (Palmaceae), planta autóctone da região, provavelmente a hospedeira original de Brassolis astyra, supondo-se uma adaptação gradual desta espécie às palmeiras exóticas introduzidas no estado como plantas ornamentais (LinK \& ALVAREZ FILHO, 1979).

Existe uma relação nítida entre disponibilidade de alimentos e a abundância de animais (RUSZCZYK, 1986). As espécies que vivem nos ecossistemas urbanos têm como característica principal uma abundância mais elevada em comparação com as comunidades naturais. As palmeiras, como são intensamente cultivadas nas cidades, representam para os herbívoros uma disponibilidade de alimento muito superior à que encontrariam na natureza. Padrões de regularidade espacial, como longas fileiras de palmeiras 
ao longo de vias públicas, por exemplo, produzem uma alta concentração de um recurso alimentar pouco frequente em condições naturais (RUSZCZYK, 1989). Espécies como Brassolis astyra produzem enormes estragos através dos estágios imaturos, deixando, muitas vezes, as plantas sem folhas nas residências, praças e parques (BIEZANKO, 1960b).

Os parâmetros analisados neste trabalho foram: morfometria das pupas e das asas de Brassolis astyra, assim como a sobrevivência e a viabilidade de ovos e pupas, longevidade das pupas, frequência de emergência de adultos, massa das pupas e dos adultos e volume das pupas.

\section{MATERIAL E MÉTODOS}

Os dados foram obtidos a partir de indivíduos amostrados em quatro localidades do Rio Grande do Sul, situadas entre os meridianos $53^{\circ} 10^{\prime} \mathrm{W}$ e $54^{\circ} 42^{\prime} \mathrm{W}$ e entre os paralelos $29^{\circ} 00^{\prime} \mathrm{S}$ e $29^{\circ} 43^{\prime} \mathrm{S}$ : Tupanciretã (TP), São Martinho da Serra (SMS), Santa Maria centro (SM) e bairro Camobi (CA). Exceto em São Martinho da Serra, as demais coletas foram realizadas em zonas urbanas, todas em Syagrus romanzoffianum (Palmaceae).

Foram investigados os seguintes parâmetros: número de ovos por posturas e índice de eclosão de ovos por postura, índice de parasitoidismo, número de ovos malogrados, posição das posturas na planta hospedeira, proporção sexual, frequência de emergência pupa-adulto, longevidade de estágios imaturos e adultos, massa e volume das pupas e morfometria das pupas e das asas dos adultos emergidos.

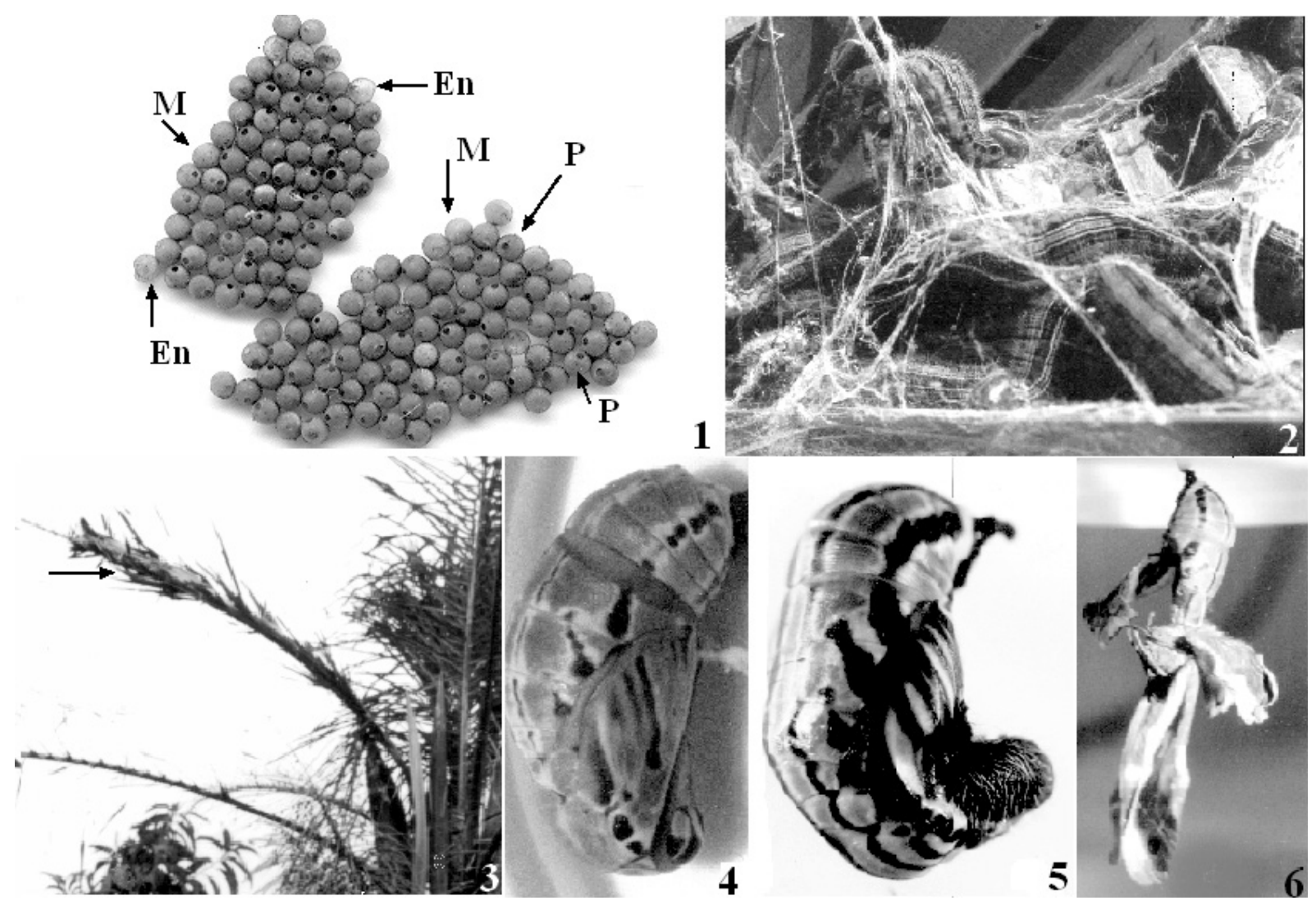

As posturas foram coletadas nos caules de Syagrus romanzoffianum, presentes nas localidades de SMS e $\mathrm{TP}$, entre um e quatro metros de altura em relação ao solo, com auxilio de uma escada, quinzenalmente, no período de março de 2006 a abril de 2007. As posturas foram acondicionadas em envelopes de papel vegetal, etiquetados e transportadas para o laboratório, para contagem de ovos e análise estatística. Nas demais localidades não foram encontradas posturas.

Ovos com o polo superior do cório comido foram registrados como eclosão normal, ovos com perfurações laterais foram considerados parasitados e ovos intactos que não eclodiram nem foram parasitados foram considerados malogrados (Fig. 1).

As larvas de quinto instar, para criação e obtenção de pupas em laboratório, foram retiradas de "cartuchos" (abrigo comunitário em forma de cone, construído por folíolos da palmeira presos com seda) (Fig. 2) em Syagrus romanzoffianum (Fig. 3) nas localidades de TP, CA e SM. Optou-se por coletar somente larvas de quinto estágio, pela determinação fácil do instar e, principalmente, devido ao longo ciclo de vida das fases de ovo até a de pupa, cerca de seis meses, minimizando assim o tempo despendido e o estresse provocado durante a criação, até a formação da pupa. As lagartas assim capturadas foram criadas em bandejas de plástico atóxico $(30 \times 40$ $\mathrm{cm}$ ), cobertas com filme P.V.C. transparente perfurado e controlada de $25^{\circ} \mathrm{C} \pm 1{ }^{\circ} \mathrm{C}, 70 \%$ de umidade e fotoperíodo de 14 horas luz. A alimentação diária das larvas foi realizada mantidas em câmara climatizada sob temperatura com folhas de Syagrus romanzoffianum, previamente

Figuras. 1-6. Brassolis astyra Godart, 1824: 1, posturas; 2, cartucho, abrigo comunitário, vista interna; 3, cartucho, vista externa; 4, pupa normal, viável; 5, pupa malformada; 6, asas não funcionais, malformação (En, ovos com eclosão normal; M, ovos malogrados; P, ovos parasitados). 
lavadas com solução de hipoclorito de sódio à $1 \%$. A vistoria e a limpeza das bandejas também foram diárias. Ao atingirem o estágio de pupa as larvas eram individualizadas em potes plásticos transparentes atóxicos $(9 \mathrm{~cm} \times 6 \mathrm{~cm})$, onde permaneciam até a eclosão do adulto. $\mathrm{O}$ volume das pupas foi estimado pelo volume de líquido deslocado, através da imersão das mesmas em água destilada dentro de uma proveta graduada.

A massa das pupas normais (Fig. 4), malformadas (Fig. 5) e dos adultos, individualmente, foi medida em balança analítica digital, com precisão até 100 gramas. Os adultos emergidos a partir das pupas foram classificados quanto à morfologia como viáveis e malformados (com asas não funcionais) (Fig. 6). O volume para cada uma das pupas foi estimado pelo volume de líquido deslocado, através da imersão das mesmas em água destilada dentro de uma proveta graduada.

As medidas efetuadas nas pupas com paquímetro foram fundamentadas em nove marcos anatômicos (laterais e ventrais) (Figs. 7, 8) originando sete diferentes medidas: comprimento longitudinal da pupa, comprimento abdominal da pupa, largura cefálica lateral, largura cefálica ventral, largura toráxica lateral, largura toráxica ventral, comprimento transversal cremaster. Os marcos anatômicos estabelecidos neste trabalho são do tipo I, segundo a classificação de BooKsteIn (1991). Após a emergência dos adultos as asas foram separadas do corpo com uma tesoura cirúrgica de ponta fina e fixadas entre folhas de material plástico transparente auto-adesivo, com dimensões aproximadas de $10 \mathrm{~cm}$ x $10 \mathrm{~cm}$. A análise morfométrica foi efetuada com base em 11 marcos anatômicos, cinco na asa anterior e seis na asa posterior (Fig. 9): comprimento e largura da asa anterior, comprimento da célula discal, comprimento e largura da asa posterior. As medidas foram realizadas com paquímetro, a partir dos marcos anatômicos marcados com alfinetes entomológicos nas asas fixadas.
Para as análises estatísticas, análise da variância, teste $t$ de Student e teste LSD (Least Square Difference), foi utilizado o programa Statistica for Windows v. 5.0. da Statsoft. As análises estatísticas foram realizadas com base nas medidas obtidas, conforme metodologia acima descrita, utilizando os dados transformados, quando não satisfaziam os critérios de normalidade e homocedasticidade.

\section{RESULTADOS E DISCUSSÃO}

Foram observados 3.284 ovos, provenientes de 11 posturas (três em TP e oito em SMS) de Brassolis astyra (média geral $=298,54 \pm 54,97$ ovos por postura), todas em Syagrus romanzoffianum (Tab. I). Dos ovos coletados em TP ( 1.036 ovos), $46 \%$ eclodiram (476 ovos), $43 \%$ estavam parasitados (443 ovos; $147,67 \pm 16,50$ ovos por postura) e $11 \%$ malograram ( 117 ovos; $39,00 \pm 57,19$ por postura). Entretanto, dos ovos coletados em SMS (2.248 ovos) apenas $4 \%$ eclodiram (93 ovos), $95 \%$ estavam parasitados ( 2.138 ovos; $267,25 \pm 46,60$ ovos por postura) e $1 \%$ malograram $(2,20 \pm 3,56$ ovos por postura). RUSZCZYK (1996) salienta que as áreas urbanas, se diferenciam das áreas naturais, por estarem organizadas em gradientes de urbanização medidos pela distância da periferia até o centro da cidade, com áreas de refúgio (parques, praças e jardins), com diferentes níveis de distúrbio humano e diferentes pressões de seleção humana específica. Os resultados obtidos demonstraram um grau de parasitismo menor em área urbana (TP), em comparação com área natural (SMS), corroborando os resultados de RUSZCZYK \& CARvalho (1993) e RuszczyK (1996) em Campinas. Segundo estes autores, as áreas urbanas proporcionam que espécies nela estabelecidas podem ter "um modelo exclusivo de escape" a parasitóides e outros inimigos naturais. Isto explicaria a abundância de brassolíneos em áreas urbanas.
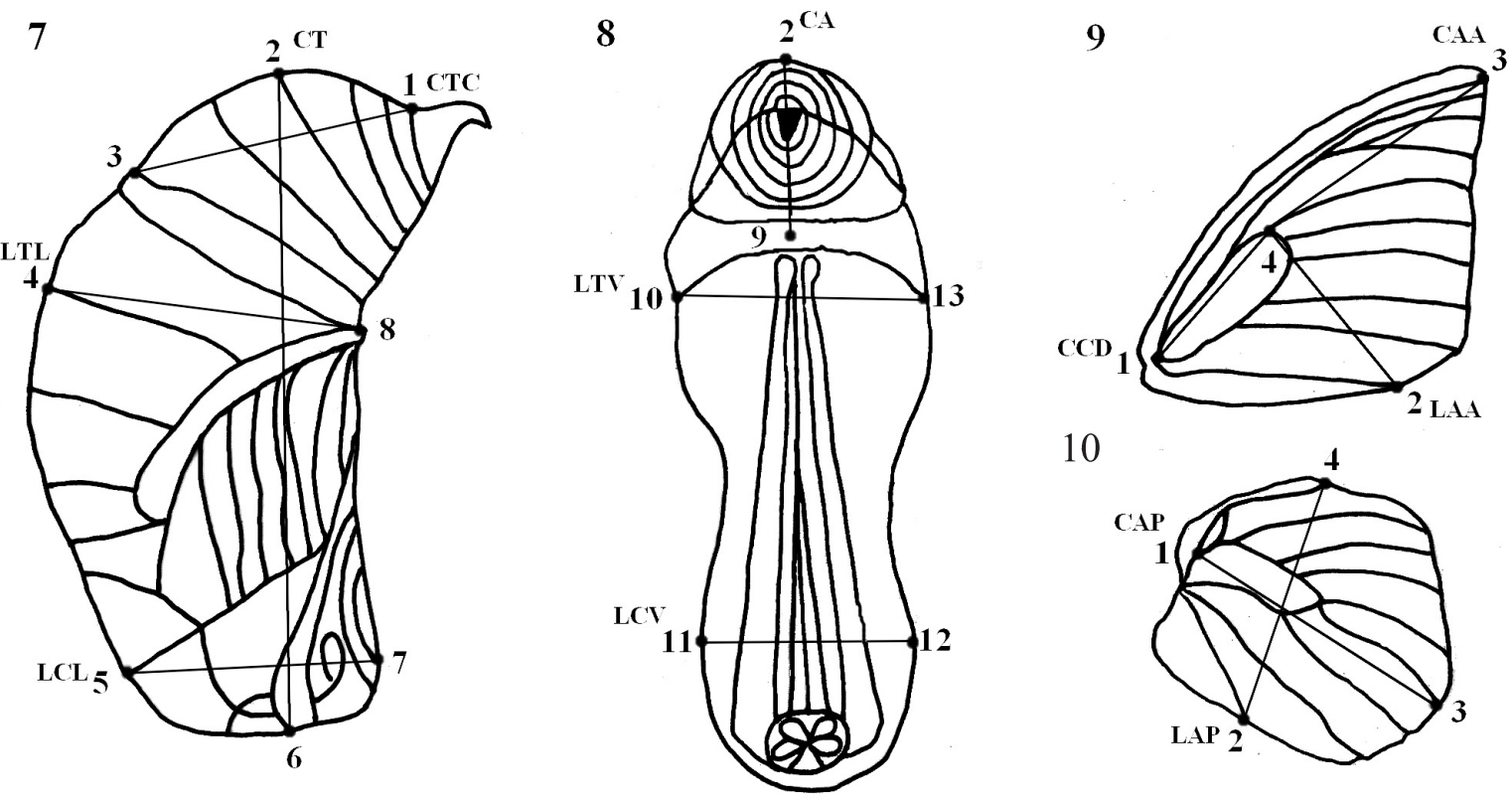

10

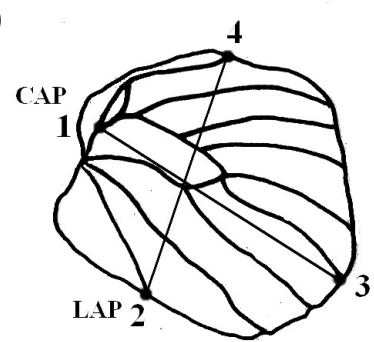

Figuras. 7-10. Brassolis astyra Godart, 1824. Marcos anatômicos utilizados nas análises das pupas: 7, vista lateral; 8, vista frontal. Marcos anatômicos utilizados nas análises das asas: 9, anterior; 10, posterior (CA (2-9), comprimento abdominal; CAA (1-3), comprimento da asa anterior; CAP (1-3), comprimento da asa posterior; CCD (1-4), comprimento da célula discal; CT (2-6), comprimento total; CTC (1-3), comprimento transversal cremaster; LAA (2-4), largura da asa anterior; LCL (5-7), largura cefálica lateral; LCV (11, 12), largura cefálica ventral; LTL (4-8), largura torácica lateral; LTV, (10-13), largura torácica ventral). 
A partir de 287 larvas coletadas em diferentes estágios, em três localidades $(\mathrm{CA}=105 ; \mathrm{SM}=22$ e TP= 160) (Tab. II), estimou-se a longevidade média das pupas. As diferenças entre os valores médios obtidos para longevidade das pupas entre as três localidades (machos, $\mathrm{CA}=18,40 \pm 1,26 ; \mathrm{SM}=15,61 \pm 1,04 \mathrm{e} \mathrm{TP}=17,25 \pm 1,48$; fềmeas, $\mathrm{CA}=19,44 \pm 3,77 ; \mathrm{SM}=15,67 \pm 1,53 \mathrm{e} \mathrm{TP}=17,22 \pm$ $2,96)$ são estatisticamente significantes $\left(\mathrm{F}_{(0,05 ; 2)}=14,966\right.$; $\mathrm{p}<0,05)$. A frequência média total de emergência de adultos foi de 0,47 (Tab. II). Entretanto, quando analisada por localidade TP apresentou o menor valor $(0,39)$ e SM o maior $(0,73)$. A proporção sexual (machos/total) estimada com base no total de indivíduos das três localidades foi de 0,54 (74 machos e 62 fêmeas). A localidade CA apresentou o menor valor $(0,44 ; 25$ machos e 32 fêmeas $)$ e SM o maior ( 0,$81 ; 13$ machos e 3 fêmeas), levando-se em conta que este valor elevado para SM possa ser decorrente do tamanho amostral pequeno. Os coeficientes de variação dos machos foram menores que os das fềmeas para as três localidades estudadas. Entretanto, o valor maior foi obtido em TP para os machos e em CA para as fêmeas. Segundo GARCIA (1989), os coeficientes de variação médios obtidos para a longevidade das pupas, provenientes das três localidades, mostram um grau de heterogeneidade média para as fêmeas $(19,41 \%)$ e baixo para os machos $(9,39 \%)$ (Tab. II). As massas e os volumes médios de pupas provenientes de SM, TP e CA, foram registrados, com base no tipo de emergência de 254 indivíduos (Tab. III). O valor

Tabela I. Número médio de ovos/postura, número médio de ovos/parasitados/postura e número de ovos malogrados de Brassolis astyra Godart, 1824, parasitando Syagrus romanzffianum (Palmaceae), gerivá, em duas localidades do RS (L, localidade; N, número de ovos/ postura; Ne, número de ovos eclodidos/postura; Nne, número de ovos não eclodidos, por causa desconhecida; Np, número de ovos parasitados/postura, não eclodidos; SMS, São Martinho da Serra; TP, Tupanciretã).

\begin{tabular}{|c|c|c|c|c|c|}
\hline Postura & $\mathrm{L}$ & $\mathrm{N}$ & $\mathrm{Ne}(\%)$ & Np (\%) & Nne (\%) \\
\hline 1 & T P & 335 & $165(49 \%)$ & $166(49 \%)$ & $4(1 \%)$ \\
\hline 2 & T P & 329 & $178(54 \%)$ & $143(43 \%)$ & $8(2 \%)$ \\
\hline 3 & T P & 372 & $133(36 \%)$ & $134(36 \%)$ & $105(28 \%)$ \\
\hline TOTAL & & 1036 & $476(46 \%)$ & $443(43 \%)$ & $117(11 \%)$ \\
\hline Média \pm d.p. & & $345,33 \pm 23,29$ & $158,67 \pm 23,16$ & $147,67 \pm 16,50$ & $39,00 \pm 57,19$ \\
\hline 4 & SMS & 336 & $4(1 \%)$ & $329(98 \%)$ & $3(1 \%)$ \\
\hline 5 & SMS & 178 & 0 & $178(100 \%)$ & 0 \\
\hline 6 & SMS & 234 & 0 & $234(100 \%)$ & 0 \\
\hline 7 & SMS & 332 & $39(12 \%)$ & $293(88 \%)$ & 0 \\
\hline 8 & SMS & 291 & $17(6 \%)$ & $274(94 \%)$ & 0 \\
\hline 9 & SMS & 301 & $3(1 \%)$ & $288(96 \%)$ & $10(3 \%)$ \\
\hline 10 & SMS & 265 & $14(5 \%)$ & 247 (93\%) & $4(2 \%)$ \\
\hline 11 & SMS & 311 & $16(5 \%)$ & $295(95 \%)$ & 0 \\
\hline TOTAL & & 2248 & $93(4 \%)$ & $2138(95 \%)$ & $17(1 \%)$ \\
\hline Média \pm d.p. & & $281 \pm 53,39$ & $11,62 \pm 13,12$ & $267,25 \pm 46,60$ & $2,20 \pm 3,56$ \\
\hline
\end{tabular}

Tabela II. Número de pupas, taxa de emergência pupa-adulto, número de machos e fêmeas, proporção sexual, tempo de duração média do estágio de pupa e morfologia dos adultos emergidos, de Brassolis astyra Godart, 1824, coletados em três localidades do RS (CA, Camobi; cv, coeficiente de variação; F, fêmeas; Fm, fêmeas malformadas; Fv, fêmeas viáveis; M, machos; Mm, machos malformados; Mv, machos viáveis; N, número de pupas; Ps, proporção sexual; SM, Santa Maria, centro; Te, taxa de emergência; Tf, tempo de duração das pupas, fêmeas; Tm, tempo de duração das pupas, machos; TP, Tupanciretã).

\begin{tabular}{lrccrcrcrrrrrr}
\hline Local & \multicolumn{1}{c}{$\mathrm{N}$} & $\mathrm{Te}$ & $\mathrm{M}$ & $\mathrm{F}$ & $\mathrm{Ps}$ & $\mathrm{Tm}$ & $\mathrm{cv}$ & $\mathrm{Tf}$ & $\mathrm{cv}$ & $\mathrm{Mv}$ & $\mathrm{Fv}$ & $\mathrm{Mm}$ & $\mathrm{Fm}$ \\
\hline CA & 105 & 0,54 & 25 & 32 & 0,44 & $18,40 \pm 1,26$ & 6,85 & $19,44 \pm 3,77$ & 19,39 & $24(96 \%)$ & $24(75 \%)$ & 1 & 8 \\
SM & 22 & 0,73 & 13 & 3 & 0,81 & $15,61 \pm 1,04$ & 6,66 & $15,67 \pm 1,53$ & 9,76 & $11(85 \%)$ & $3(100 \%)$ & 2 & 0 \\
T P & 160 & 0,39 & 36 & 27 & 0,57 & $17,25 \pm 1,48$ & 8,58 & $17,22+2,96$ & 17,19 & $22(61 \%)$ & $21(78 \%)$ & 14 & 6 \\
\hline TOTAL & 287 & 0,47 & 74 & 62 & 0,54 & $17,35 \pm 1,63$ & 9,39 & $18,29 \pm 3,55$ & 19,41 & $57(77 \%)$ & $48(77 \%)$ & $17(23 \%)$ & $14(23 \%)$ \\
\hline
\end{tabular}

Tabela III. Massa e volume das pupas de Brassolis astyra Godart, 1824 que emergiram adultos viáveis, malformados ou que não ocorreu emergência (mortas), procedentes de três localidades do RS e mantidas sob condições de laboratório $\left(25^{\circ} \mathrm{C} \pm 1{ }^{\circ} \mathrm{C}, 70 \%\right.$ de umidade e fotoperíodo de 14 horas luz) (CA, Camobi; cv, coeficiente de variação; Mp, massa da pupa; P, procedência; SM, Santa Maria, centro; Tem, tipo de emergência dos adultos; TP, Tupanciretã; Vp, Volume da pupa).

\begin{tabular}{llrrrrr}
\hline P & Tem & N & Mp $(\mathrm{g})$ & cv $(\%)$ & Vp $(\mathrm{ml})$ & $\mathrm{cv}(\%)$ \\
\hline SM & Viável & 14 & $1,97 \pm 0,40$ & 20,30 & $2,16 \pm 0,25$ & 11,57 \\
SM & Morta & 7 & $1,95 \pm 0,16$ & 8,20 & $2,00 \pm 0,29$ & 14,50 \\
SM & Malformada & 1 & $1,95 \pm 0,00$ & 0 & $2,00 \pm 0,00$ & 0 \\
CA & Viável & 24 & $3,03 \pm 0,51$ & 16,83 & $3,14 \pm 0,63$ & 20,06 \\
CA & Morta & 64 & $2,74 \pm 0,57$ & 20,80 & $2,91 \pm 0,67$ & 23,02 \\
CA & Malformada & 7 & $1,99 \pm 0,37$ & 18,59 & $2,40 \pm 0,39$ & 16,25 \\
T P & Viável & 4 & $1,90 \pm 0,35$ & 18,42 & $2,12 \pm 0,25$ & 11,79 \\
T P & Morta & 99 & $1,52 \pm 0,49$ & 32,24 & $1,89 \pm 0,64$ & 33,86 \\
T P & Malformada & 34 & $1,42 \pm 0,38$ & 26,76 & $1,76 \pm 0,49$ & 27,84 \\
\hline TOTAL & & 254 & $2,02 \pm 0,78$ & 38,61 & $2,29 \pm 0,79$ & 34,53
\end{tabular}


médio da massa das pupas, das quais emergiram adultos viáveis, foi maior que das pupas das quais emergiram adultos e daquelas que emergiram borboletas malformadas. O volume médio das pupas das quais emergiram adultos viáveis também foi maior que das pupas onde não emergiram adultos e daquelas que emergiram borboletas com asas malformadas. Portanto, embora os valores médios obtidos para indivíduos provenientes de diferentes localidades sejam diferentes, tanto para o valor médio da massa quanto para o volume médio das pupas, as médias das pupas das quais não emergiram adultos ou emergiram com malformações são representadas pelos valores mais baixos que os obtidos para as pupas que originaram adultos viáveis (com asas funcionais). As diferenças entre as massas e volumes médios obtidos entre as três localidades, de acordo com o tipo de emergência das pupas, são estatisticamente significativas $\left(\mathrm{F}_{\text {(peso) }}=23,83 ; 2 \mathrm{gl} ; \mathrm{p}<0,05 ; \mathrm{F}_{\text {(volume) }}=14,35\right.$; $2 \mathrm{gl} ; \mathrm{p}<0,05)$. Os coeficientes de variação, tanto para a massa como para o volume das pupas (Tab. III), indicam um grau de heterogeneidade média para as três localidades. Os valores médios obtidos para as pupas das três localidades igualmente são significativos tanto para a massa $\left(\mathrm{F}_{(0.05 ; 2)}=171,59 ; \mathrm{p}<0,05\right)$ quanto para o volume $\left(\mathrm{F}_{(0,05 ; 2)}=89,01 ; \mathrm{p}<0,05\right)$. Com base nos adultos emergidos a partir das pupas (Tab. II) verificou-se que $77 \%$ dos machos e das fêmeas apresentaram uma morfologia normal (viável) com asas funcionais e 23\% eram malformadas. As diferenças verificadas em ambos os sexos, quanto à proporção de indivíduos viáveis e malformados não são significativas, quando analisadas através de um teste de contingência $\left(\chi_{(0,05)}^{2}=0,0029\right)$. Embora os indivíduos malformados possam ser associados a valores médios menores de massa, não pode ser descartada a hipótese de que parte dessa malformação seja atribuída ao estresse de manipulação em laboratório.

A maior parte dos modelos evolutivos presume que as proporções alométricas procedem de seleção estabilizadora que atua no tamanho corporal como um todo (Gould, 1966). Assim, as relações entre área e tamanho de determinadas estruturas do corpo são constantes e muitas relações orgânicas só podem ser mantidas por alteração da forma (Gould, 1966). Segundo SCoble, (1992), a função primária da fase de pupa em insetos holometabolos é possivelmente permitir expansão das asas. Deste modo, seleção diferencial atuando em determinadas partes do corpo pode determinar alteração na forma (LANDE, 1979; Zeng, 1988; RiskA, 1989). Assim sendo, a formação de asas não funcionais, poderia estar relacionada a alterações no tamanho da pupa, decorrentes da perda de coadaptações relacionadas com a reorganização, crescimento e diferenciação que ocorre nesta fase, associadas a valores extremos (maiores ou menores) de massa e volume das pupas em relação aos valores médios ótimos.

As medidas lineares realizadas nas pupas foram baseadas em sete marcos anatômicos. As diferenças entre os valores médios estimados entre machos e fêmeas para seis das sete medidas realizadas são estatisticamente significantes (Tab. IV), exceto para o comprimento abdominal, tanto para as pupas das quais emergiram indivíduos viáveis, quanto para as pupas que não emergiram borboletas ou emergiram adultos malformados. O coeficiente de variação obtido para todos os parâmetros estimados é semelhante em ambos os sexos, indicando um grau de heterogeneidade média.

Os resultados obtidos mostram um dimorfismo sexual de tamanho e forma, entre as pupas das fêmeas e dos machos; sugerem também que o tamanho e a forma das pupas podem determinar a viabilidade em gerar adultos com asas funcionais ou não. Tal fato pode estar associado a uma seleção estabilizadora, cuja massa e forma estariam associados a valores médios ótimos. Embora a procedência dos indivíduos analisados seja de locais diferentes (Tab. III), os parâmetros obtidos que determinam o tamanho (massa) e a forma (volume) estão na mesma direção quando comparamos ambos os sexos.

As medidas realizadas nas asas dos adultos viáveis emergidos estão baseadas em onze marcos anatômicos (Fig. 9). Os valores estimados para as medidas realizadas nas asas anteriores, posteriores e célula discal são maiores nas fêmeas (Tab. V). Os coeficientes de variação para as asas anteriores das fêmeas são maiores que os

Tabela IV. Morfologia dos adultos de Brassolis astyra Godart, 1824 emergidos de pupas criadas sob condições de laboratório $\left(25^{\circ} \mathrm{C} \pm 1{ }^{\circ} \mathrm{C}\right.$, $70 \%$ de umidade e fotoperíodo de 14 horas luz) (CA, comprimento abdominal; CT, comprimento total; CTC, comprimento tórax/ cremaster; cv, coeficiente de variação; F, fêmeas; LCL, largura cefálica lateral; LCV, largura cefálica ventral; LTL, largura toráxica lateral; LTV, largura toráxica ventral; M, machos; PE, parâmetros estimados).

\begin{tabular}{|c|c|c|c|c|c|c|c|}
\hline $\mathrm{PE}(\mathrm{mm})$ & Sexo & Viável & $\mathrm{cV}(\%)$ & Morta & $\mathrm{cV}(\%)$ & Malformada & $\mathrm{cV}(\%)$ \\
\hline $\mathrm{CT}$ & $\mathrm{F}$ & $29,01 \pm 2,45$ & 8,44 & $27,38 \pm 2,74$ & 10,01 & $25,80 \pm 1,43$ & 5,54 \\
\hline $\mathrm{CT}$ & M & $26,91 \pm 2,56$ & 9,51 & $25,38 \pm 3,55$ & 14,00 & $22,85 \pm 1,30$ & 5,69 \\
\hline $\mathrm{CA}$ & $\mathrm{F}$ & $9,70 \pm 0,70$ & 7,22 & $8,73 \pm 1,77$ & 20,27 & $6,78 \pm 1,45$ & 21,39 \\
\hline $\mathrm{CA}$ & M & $8,74 \pm 0,70$ & 8,01 & $8,27 \pm 1,07$ & 12,94 & $7,02 \pm 1,80$ & 25,64 \\
\hline $\mathrm{LCL}$ & $\mathrm{F}$ & $10,63 \pm 1,03$ & 9,69 & $10,38 \pm 1,18$ & 11,37 & $10,02 \pm 0,94$ & 9,38 \\
\hline $\mathrm{LCL}$ & M & $10,61 \pm 1,05$ & 9,90 & $9,67 \pm 1,04$ & 10,75 & $9,30 \pm 0,94$ & 10,11 \\
\hline $\mathrm{LCV}$ & $\mathrm{F}$ & $11,26 \pm 1,01$ & 8,97 & $10,82 \pm 1,37$ & 12,66 & $9,88 \pm 0,67$ & 6,78 \\
\hline $\mathrm{LCV}$ & $\mathrm{M}$ & $10,52 \pm 1,02$ & 9,69 & $9,82 \pm 0,97$ & 9,88 & $9,30 \pm 0,64$ & 6,88 \\
\hline LTL & $\mathrm{F}$ & $13,57 \pm 1,35$ & 9,95 & $13,01 \pm 1,75$ & 13,45 & $12,25 \pm 1,69$ & 13,79 \\
\hline LTL & M & $12,21 \pm 0,81$ & 6,63 & $11,26 \pm 1,24$ & 11,01 & $11,13 \pm 1,51$ & 13,57 \\
\hline LTV & $\mathrm{F}$ & $13,16 \pm 1,18$ & 8,97 & $12,33 \pm 1,17$ & 9,49 & $12,15 \pm 1,49$ & 12,26 \\
\hline LTV & $\mathrm{M}$ & $12,00 \pm 0,81$ & 6,75 & $10,92 \pm 1,24$ & 11,35 & $10,40 \pm 1,51$ & 14,52 \\
\hline CTC & $\mathrm{F}$ & $12,42 \pm 1,02$ & 8,21 & $11,62 \pm 1,41$ & 12,13 & $12,23 \pm 0,81$ & 6,62 \\
\hline CTC & M & $11,06 \pm 0,78$ & 7,05 & $10,46 \pm 1,36$ & 13,00 & $10,70 \pm 1,06$ & 9,91 \\
\hline
\end{tabular}


Tabela V. Medidas morfometricas das asas anteriores e posteriores de machos e fêmeas de Brassolis astyra Godart, 1824, criadas sob condições de laboratório $\left(25^{\circ} \mathrm{C} \pm 1{ }^{\circ} \mathrm{C}, 70 \%\right.$ de umidade e fotoperíodo de 14 horas luz) (CAA, comprimento da asa anterior; CAP, comprimento da asa posterior; $\mathrm{CCD}$, comprimento da célula discal; cv, coeficiente de variação; LAA, largura da asa anterior; LAP, largura da asa posterior; n, número de indivíduos; PE, parâmetros estimados).

\begin{tabular}{|c|c|c|c|c|c|c|}
\hline PE (mm) & Machos & cv $(\%)$ & $\mathrm{n}$ & Fêmeas & $\mathrm{cv}(\%)$ & $\mathrm{n}$ \\
\hline CAA & $37,56 \pm 3,737$ & 9,95 & 25 & $47,53 \pm 4,19$ & 10,08 & 16 \\
\hline LAA & $20,27 \pm 2,500$ & 12,33 & 26 & $24,80 \pm 3,06$ & 12,34 & 16 \\
\hline $\mathrm{CCD}$ & $19,01 \pm 2,885$ & 15,18 & 26 & $25,12 \pm 2,44$ & 9,71 & 16 \\
\hline CAP & $27,50 \pm 3,076$ & 11,18 & 26 & $37,31 \pm 3,45$ & 9,25 & 16 \\
\hline LAP & $25,00 \pm 2,793$ & 11,17 & 26 & $29,79 \pm 3,20$ & 10,74 & 15 \\
\hline
\end{tabular}

dos machos, entretanto, para a célula discal e asas posteriores, os valores obtidos mostram uma maior variação nos machos. A diferença entre as médias de machos e fêmeas analisadas através do teste LSD mostra que as diferenças entre as médias obtidas são estatisticamente significantes entre os sexos.

Com base nos estudos de Di Mare \& Corseuil (2004a, 2004b) e Di MARE et al. (2004), em Papilioninae, podemos inferir três explicações para a ocorrência de dimorfismo sexual em Brassolis astyra: a primeira é que fêmeas com maior tamanho do corpo podem ser favorecidas com a produção de maior volume e tamanho das posturas; a segunda seria devido a diferentes taxas de alocação de energia para crescimento e reprodução entre os sexos; ou o dimorfismo sexual pode ser um mecanismo de redução da competição intra-específica por recursos alimentares.

Os dados referentes à longevidade e mortalidade de pupas e adultos mostram que os valores estimados podem ser influenciados pela variabilidade e ecologia da população local de onde as larvas foram retiradas, já que em laboratório estavam submetidas às mesmas condições de criação.

Duas explicações podem estar relacionadas à ocorrência de dimorfismo sexual em Brassolis astyra. A primeira é que fêmeas com maior tamanho do corpo podem ser favorecidas com a produção de maior volume e tamanho da prole e a segunda seria devido a diferentes taxas de alocação de energia para crescimento e reprodução entre os sexos já que os adultos não se alimentam.

Agradecimentos. Aos pesquisadores Alexandre Ruszczyk, Simone Mundstock Jahnke e Flávio Roberto Mello Garcia pelas críticas e sugestões realizadas na revisão deste artigo.

\section{REFERÊNCIAS BIBLIOGRÁFICAS}

Biezanko, C. M. 1960a. IV. Satyridae, Morphidae et Brassolidae da zona Sueste do Rio Grande do Sul. Arquivos de Entomologia, Série A:1-13.

1960b. IV. Satyridae, Morphidae et Brassolidae da zona Missioneira do Rio Grande do Sul. Arquivos de Entomologia, Série B:1-10.

Bookstein, F. L. 1991. Morphometric tools for landmark data: Geometry and Biology. Cambridge, Cambridge University. 455p.

Di mare, R. A. \& Corseuil, E. 2004a. Morfometria de papilionídeos (Lepidoptera; Papilionidae) ocorrentes em quatro localidades do Rio Grande do Sul, Brasil: II. Relação entre as partes do corpo, aerodinâmica de vôo e tipos de asas. Revista Brasileira de Entomologia 21(4):833-846.

2004b. Morfometria de papilionídeos (Lepidoptera; Papilionidae) ocorrentes em quatro localidades do Rio Grande do Sul, Brasil: III. Análise da forma das asas através de marcos anatômicos. Revista Brasileira de Entomologia 21(4):833-846.

Di mare, R. A.; Schwartz, G. \& Corseuil, E. 2004. Morfometria de papilionídeos (Lepidoptera; Papilionidae) ocorrentes em quatro localidades do Rio Grande do sul: I. Comparações com a massa. Revista Brasileira de Entomologia 48(4):535-545.

GarCiA, C. H. 1989. Tabelas para classificação de coeficientes de variação. Piracicaba, Instituto de Pesquisas e Estudos Florestais (circular técnica, 171). 12p.

Gould, S. J. 1966. Allometry and size in ontogeny and phylogeny. Biological Review 41:587-640.

LANDE, R. 1979. Quantitative genetic analysis of multivariate evolution applied to brain: body size allometry. American Naturalist 33:402-416.

Link, D. \& Alvarez Filho, A. 1979. Palmeiras atacadas por lagartas de Brassolidae (Lepidoptera) em Santa Maria, RS. Revista do Centro Ciências Rurais 9(2):221-225.

Link, D.; Biezanko, C. M.; Carvalho, S. \& Tarragó, M. F. S. 1980. Lepidoptera de Santa Maria e Arredores. III. Morphidae e Brassolidae. Revista do Centro de Ciências Rurais 10(2): 191-195.

RiskA, B. 1989. Composite traits, selection response, and evolution. Evolution 43:1172-1191.

Ruszczyk, A. 1986. Hábitos Alimentares de Borboletas Adultas e sua Adaptabilidade ao Ambiente Urbano. Revista Brasileira de Biologia 46(2):419-427.

1989. Lagarta-de-Palmeira: Nossa Amiga ou Inimiga? Revista Ciência Hoje 10(58):70-71.

1996. Spatial Patterns in Pupal Mortality in Urban Palm Caterpillars. Oecologia 107:356-363.

RuszczyK, A. \& Carvalho JR., M. C. 1993. Malfuction of Ecdysis and Female Biased Mortality in Urban Brassolis sophorae (Nymphalidae: Brassolinae). Journal of the Lepidopterists Society 47(2):134-139.

Scoble, J. M. 1992. The Lepidoptera: form, function and diversity. Oxford, Oxford University. 404p.

ZENG, Z. 1988. Long-term correlated responses, interpopulation covariation and interspecific allometry. Evolution 42:363-374.

Recebido em julho de 2007. Aceito em novembro de 2008. ISSN 0073-4721

Artigo disponível em: www.scielo.br/isz

Iheringia, Sér. Zool., Porto Alegre, 99(2):194-199, 30 de junho de 2009 